\title{
The Hippocampus and Long-Term Object Memory in the Rat
}

\author{
Norbert Vnek and Lawrence A. Rothblat \\ Department of Psychology, George Washington University, Washington, DC 20052
}

Animal models of amnesia have yielded many insights into the neural substrates of different types of memories. Some very important aspects of memory, however, have been ignored in research using experimental animals. For example, to examine long-term memory investigators traditionally have relied on measures of information acquisition, which stand in contrast to the measures of retention commonly used in work with humans. We have recently developed a behavioral paradigm that measures both the acquisition and long-term retention of object discriminations, and found a selective retention impairment in rats with entorhinal-hippocampal disconnection (Vnek et al., 1995).

The present study was designed to determine whether direct damage to the hippocampus likewise would lead to a selective deficit in the retention of visual discriminations. Rats with aspi- ration lesions of the dorsal hippocampus, rats with neocortical control lesions, and normal controls were trained on three object discrimination problems and then retrained 3 weeks later to measure retention. All animals showed the same level of performance during the training (acquisition) phase of testing, but the performance of animals with dorsal hippocampal injury fell below that of controls during retraining (retention). Taken together, these and our earlier results suggest that the hippocampus and anatomically related structures are particularly important for retaining visual discriminations over long delay intervals. These findings may clarify the role of the hippocampus in nonspatial memory.

Key words: acquisition; retention; amnesia; nonspatial memory; entorhinal cortex; hippocampus
The medial portion of the temporal lobe is important for memory (for review, see Murray, 1992; Squire, 1992; Jarrard, 1993; ZolaMorgan and Squire, 1993; Mishkin and Murray, 1994). Evidence of this comes from studies of human clinical populations with amnesia and known or suspected medial temporal pathology (Scoville and Milner, 1957; Zola-Morgan et al., 1986; Braak and Braak, 1993). However, because of the rarity of circumscribed lesions in human patients, animal models of amnesic disorders have been developed so that more precise functional neuroanatomical studies are possible.

Perhaps the most widely modeled amnesic patient is H. M., who underwent bilateral medial temporal resection in 1953 and whose consequent memory functions and dysfunctions have since been studied intensively (Scoville and Milner, 1957). H. M.'s memory appears normal within the parameters of short-term memory, but is typically grossly impaired when he is called on to remember information in excess of those parameters (Wickelgren, 1968). Impaired long-term memory in the face of normal short-term memory is in fact a defining feature of medial temporal amnesia. Thus, to examine medial temporal amnesia in both human patient populations and experimental animals, it is imperative to assess long-term memory functions.

Behavioral paradigms used to measure memory in animal models commonly use visual discriminations, usually objects, as stimuli. The validity of these tasks can be assessed by comparing the performance of lesioned animals to that of human amnesics on analogous memory tests. When given extended

Received Oct. 26, 1995; revised Jan. 4, 1996; accepted Jan. 8, 1996.

This work was supported by Alzheimer's Association Grant PRG-94-172.

Correspondence should be addressed to Dr. Lawrence A. Rothblat, Department of Psychology, George Washington University, Washington, DC 20052.

Dr. Vnek's present address: Section of Neurobiology, Yale University School of Medicine, New Haven, CT 06510.

Copyright (C) 1996 Society for Neuroscience $0270-6474 / 96 / 162780-08 \$ 05.00 / 0$ time (i.e., $15-20 \mathrm{sec}$ ) to study a set of pictures, H. M. can perform at the level of controls on certain memory tests (e.g., yes/no recognition judgments) after delay intervals of up to $1 \mathrm{~d}$, but his performance drops dramatically when longer intervals ( 1 week or more) are used (Huppert and Piercy, 1979; Freed and Corkin, 1988). These results underscore the importance of measuring extended delay intervals in behavioral paradigms used with experimental animals.

Although some animal models of amnesia have measured longterm memory, the tasks used to do so typically depart from those administered to human clinical populations in some very important respects. In studies with humans, subjects are shown a list of stimuli, a delay interval is imposed, and a test of retention (recall or recognition) follows. In contrast, with the exception of a few early studies (Orbach et al., 1960; Correll and Scoville, 1965), investigations of long-term memory in experimental animals have rarely used behavioral paradigms that assess both the acquisition and retention of the same set of stimuli. For example, a measure of long-term nonspatial memory commonly used in animal models of amnesia is the concurrent object discrimination task, in which the dependent variable is the amount of training needed to reach criterion on several discrimination problems (Moss et al., 1981; Zola-Morgan and Squire, 1985). This task is to a large extent an index of information acquisition, and as such is more a measure of learning than memory.

We have recently developed a behavioral paradigm that measures both the acquisition and long-term retention of visual discrimination problems in rats (Vnek et al., 1995). On this task, rats with entorhinal-hippocampal disconnection from angular bundle transections show normal acquisition but impaired retention over a 2 week interval. The present study was conducted to determine whether direct damage to the hippocampus would likewise lead to a selective retention deficit. 


\section{MATERIALS AND METHODS}

Subjects. The subjects were 18 male Wistar rats (Charles River, Wilmington, MA). All animals were $-120 \mathrm{~d}$ old at the start of the experiment and weighed between 400 and $500 \mathrm{gm}$. Animals were fooddeprived to $85 \%$ of normal body weight during behavioral testing.

Dorsal hippocampal lesions were chosen instead of removing the entire hippocampus because recent studies have shown that the size of dorsal hippocampal lesions in rats is directly related to spatial memory impairments whereas the size of ventral hippocampal lesions is not (Moser et al., 1993, 1995). Based on the assumption that the relative importance of the dorsal hippocampus can be extended to nonspatial memory, the lesions of the present study were intended to involve only this division of the hippocampus. Dorsal hippocampal lesions also can be achieved without damaging rhinal (i.e., entorhinal and perirhinal) cortex, which principally surrounds the ventral hippocampus in the rat. Avoiding damage to this area is critical because lesions limited to rhinal cortex have been found to lead to nonspatial memory impairments in both monkeys (Gaffan and Murray, 1992; Meunier et al., 1993) and rats (Otto and Eichenbaum, 1992; Bunsey and Eichenbaum, 1993; Mumby and Pinel, 1994).

Surgery. Nine rats received bilateral aspiration lesions of the dorsal hippocampus, 5 rats served as unoperated controls, and 4 rats received lesions controlling for the cortical damage included in hippocampal lesions.

During surgery, animals were anesthetized with a mixture of ketamine $(90 \mathrm{mg} / \mathrm{kg})$ and xylazine $(15 \mathrm{mg} / \mathrm{kg})$, administered by intraperitoneal injection. Surgery utilized a stereotaxic device, with the rat's head in a level position for all surgical procedures, and was visually guided using a surgical microscope.

Under anesthesia, animals were placed in the stereotaxic device, an incision was made in the skin overlaying the dorsal surface of the skull, and the skin was retracted to expose the skull. For dorsal hippocampal lesions (group DH), bilateral triangular bone flaps over the dorsal part of the hippocampus were removed. A cranial opening in the form of a triangle was chosen because it optimizes access to the dorsal hippocampus as it expands laterally along its septotemporal axis.

Bore holes were drilled at three points on the skull, and straight lines connecting these points were made. The single anterior point of the bone flap was made $-2.2 \mathrm{~mm}$ from bregma and $1.0 \mathrm{~mm}$ lateral to the midline. The two posterior points were $-4.5 \mathrm{~mm}$ from bregma, with one point 1 $\mathrm{mm}$ lateral to the midline and the other point $4.0 \mathrm{~mm}$ lateral to the midline. After craniotomy and laceration of the dura, neural tissue was aspirated as defined by the triangular bone flap to a depth of $\sim 2.5 \mathrm{~mm}$ below dura. A small plastic collar was attached to the aspiration cannula to limit the depth of insertion. The collar was glued $3.5 \mathrm{~mm}$ from the tip of the cannula, which accounted for the $1 \mathrm{~mm}$ of space between the top of the skull and the dorsal surface of the brain. The procedure was intended to remove frontal (rostrally) and occipital (caudally) neocortex and the underlying splenium of the corpus callosum, as well as the CA1CA3 fields of the hippocampus, the lateral portion of the subiculum, and the lateral division of the dentate gyrus in the dorsal hippocampus.

Dorsal hippocampal control lesions (DHC) were also guided by bilateral triangular bone flaps. The coordinates and craniotomy procedure for DHC lesions were identical to those used for DH lcsions, although the former were only extended to a depth of $\sim 1.0-1.5 \mathrm{~mm}$ below dura. A plastic collar was fitted onto the aspiration cannula, $2.0 \mathrm{~mm}$ from its tip, to control insertion and lesion depth. DHC lesions were intended to remove frontal neocortex rostrally and occipital neocortex caudally. The callosal and cingulum fibers damaged by DH lesions were also intended to be removed, although only to the extent that subjacent alvear fibers and hippocampal cellular fields could be spared.

After surgical procedures, gel foam soaked in physiological saline was placed in the lesion cavities, the incised skin was sutured and sterilized, and the animal was placed on a heating pad set to $37^{\circ} \mathrm{C}$ until emerging from anesthesia. A 2 week recovery period followed surgery.

Rats in the normal control group (NC) did not undergo any surgical procedures.

Materials. The object discrimination task was conducted on a wood Y-Maze as described previously (Vnek et al., 1995). The maze was constructed of a $90 \times 9 \mathrm{~cm}$ runway, which divided at a $30^{\circ}$ angle into two $25 \times 7 \mathrm{~cm}$ goal arms. At the threshold of each goal arm was a ridge $7 \mathrm{~cm}$ in length, $1 \mathrm{~cm}$ in height, and $1 \mathrm{~cm}$ in width, which served as a physical demarcation of the entrance of the goal arm. Ten centimeters beyond each ridge on the goal arm laid a $10 \times 7 \times 0.5 \mathrm{~cm}$ insert with a hole $2 \mathrm{~cm}$ in diameter that served as a food well. The center of the food well was positioned $13 \mathrm{~cm}$ behind the entrance ridge. Around the front (facing the ridge and runway) of the food well, five small holes were drilled into the insert, each of which contained one $45 \mathrm{mg}$ food pellet (Noyes, Lancaster, NH). These pellets were used to mask the smell of the food pellets used to reward animals during object discrimination testing so that olfactory cues could not be used to learn the discriminations. Between trials, animals were restrained from traversing the runway with a removable 40 $\times 20 \mathrm{~cm}$ free standing door, which was placed $25 \mathrm{~cm}$ from the beginning of the runway.

Procedure. On the object discrimination task, rats were trained on three successive object discrimination problems and then later retrained on these discriminations to measure retention. The protocol for behavioral testing was identical to that of Vnek et al. (1995), except that a 3 week delay interval was used in the present study rather than the 2 week interval of nur earlier study. For a given problem, each object from the test pair was placed on the food well of one of the goal arms of the Y-maze, although food pellets were placed only in the well that held the predetermincd correct object. At this time, the animal was placed at the beginning of the runway and was prevented from traversing the runway by the restraining door. To start a trial, the door was removed and the animal was allowed to navigate the runway and enter a goal arm of his choosing. The rat was then allowed to displace the object covering the food well of the goal arm he chose. A choice, whether correct or incorrect, was determined to have been made when the animal's front paws crossed the ridge demarcating the entrance of one of the goal arms. The placement of the stimuli (both correct and incorrect objects) on the arms of the maze (i.e., the left/right arm assignment) was randomized to preclude use of spatial cues in learning the problems. After a choice was made, the animal was returned to the beginning of the runway, and the restraining door was replaced until the start of the next trial. The intertrial interval was $\sim 10 \mathrm{sec}$.

Animals were given 20 trials of training each day, with a correction procedure that called for repeating a particular trial until the correct object was displaced, and training occurred $5 \mathrm{~d}$ per week. Animals were trained to a criterion of $90 \%$ correct responses (i.e., at least 18 correct first-response trials on a particular day), with the dependent measure being the number of days required to reach criterion. Training on the second and third problems immediately followed criterion performance on the preceding problem. There were three different orders in which animals received training on the individual pairs of objects; these orders were counterbalanced across groups.

After reaching criterion on the third discrimination problem, a 3 week interval was imposed, during which animals were not tested in any way. Animals then received retraining on the three object discrimination problems. The procedure used during the retraining phase of testing was identical to that of training, except that a $15 \mathrm{~d}$ limit on testing was imposed for each problem.

Histology. For histological verification of the lesions, DH and DHC animals were perfused after completion of behavioral testing. Animals were fully anesthetized via intraperitoneal injection of Nembutal and transcardially perfused, first with $100 \mathrm{ml}$ of rinse solution (1\% paraformaldehyde in $0.1 \mathrm{M}$ phosphate buffer, $\mathrm{pH} 7.4$ ) and then with $300 \mathrm{ml}$ of buffered $4 \%$ paraformaldehyde, $\mathrm{pH} 7.4$. After perfusion, the brain was removed, post fixed in $4 \%$ buffered paraformaldchydc for $3 \mathrm{hr}$, and then equilibrated in a $20 \%$ buffered sucrose solution. All brains were then frozen and $32 \mu \mathrm{m}$ coronal sections were taken with a cryostat at $-13^{\circ} \mathrm{C}$. Every eighth section was retained and later stained with cresyl violet to determine the integrity of the target structures. Verification of lesions was achieved through comparison of cresyl violet-stained material from animals in the lesion groups to the normative representations in the stereotaxic atlas of the rat brain of Paxinos and Watson (1986).

Cresyl violet-stained coronal sections taken from $\mathrm{DH}$ brains revealed homogeneous, symmetrical, and appropriately positioned bilateral lesions. A schematic illustration of a representative dorsal hippocampal lesion appears in Figure 1. Most of the lesions began near the rostral pole of the hippocampus, although several started more rostrally, with the fimbria. Of the latter, the damage to the fimbria in two cases was bilateral and, in one case, unilateral. The lesion of rostral hippocampus was complete in both hemispheres in three rats, in which there was total removal of the entire dentate gyrus (i.e., infrapyramidal blade, suprapyramidal blade, hilus), and Ammon's horn (i.e., fields CA1-CA3), as well as bilateral damage to overlying retrosplenial and frontal cortices (Fig. 2). Unintended damage to the dorsal thalamus was evident in two of these animals. Of the animals with incomplete lesions of the hippocampus, most showed bilateral elimination of lateral CA1, the entire CA2 field, 
Figure 1. Schematic diagram of a representative dorsal hippocampal lesion. Four coronal sections are shown to depict the rostrocaudal extent of the lesion. Note the sparing of medial Ammon's horn and dentate gyrus rostrally, and lateral hippocampal formation caudally. Numbers represent distance from bregma. Adapted with permission from Paxinos and Watson (1986) (their Figs. 23, 31, 39, 47).

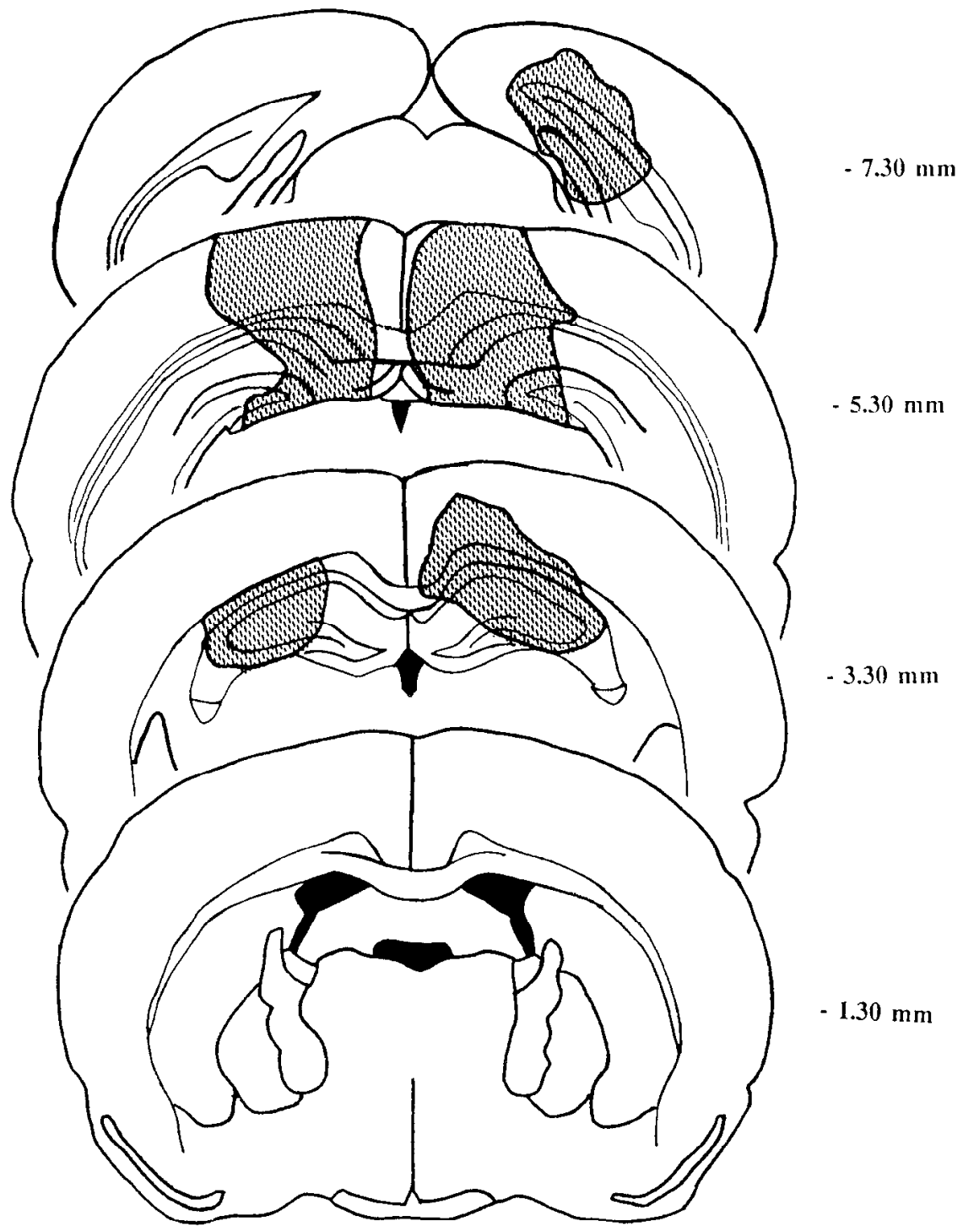

lateral CA3, and lateral aspects of the dentate gyrus. Material from two cases showed a similar lesion at this level unilaterally, with one brain revealing only moderate damage to the CA1 field in the other hemisphere and the other showing no lesion in the contralateral hippocampus. The lesion in one animal began rather caudally, and no hippocampal involvement was evident at the level of the rostral third of the hippocampus. Animals with incomplete lesions of the rostral hippocampus had retrosplenial cortical and frontal cortical involvement similar to that seen in those with complete lesions.

More caudally, beginning at the level of the hippocampal flexure, damage to the hippocampus was a bit more variant. Bilateral damage to the subiculum and dorsal CA1 field was evident in seven brains, with the remaining two showing similar damage unilaterally. The contralateral hippocampus of the latter animals was largely intact, although the overlying dorsal hippocampal commissure was damaged in both. At this level, many of the lesions extended ventrally to include the dentate gyrus, although dentate involvement was usually confined to its medial aspect. There was little thalamic involvement at this level. In addition, most animals also had concomitant bilateral damage to overlying retrosplenial and occipital cortices (principally occipital area $2 \mathrm{ML}$ ). Further caudally, near the caudal pole of the hippocampus, there was little evidence of the lesion. At this level, the only consistent sign of pathology was gliosis in the white matter of the angular bundle. One animal, however, sustained bilateral damage to dorsal presubiculum, and two animals had unilateral damage to this structure.

Like the experimental lesions, aspiration lesions of neocortex superjacent to the dorsal hippocampus were very much as intended. Histological material revealed elimination of all layers of neocortical tissue, as well as subjacent white matter, at lesion sites. The lesions first appeared in neocortex above the area of the rostral pole of the hippocampus in two cases and in the two others began at the level of the fimbria and ventral hippocampal commissure. Control lesions extended to neocortex above the caudal hall of the hippocampus bilaterally, but stopped well before the caudal pole of the hippocampus. One case proved to be rather limited in size on the rostrocaudal plane bilaterally, and was not evident until the level of the hippocampal Hexure. Control lesions also tended to be larger and more medial in the right hemisphere, where removal of retrosplenial cortex was apparent, as well as the frontal (rostrally) and occipital (caudally) cortices adjacent to it. On the left side, cortical damage was largely confined to frontal cortex at rostral levels and occipital cortex at caudal levels, with little retrosplenial involvement. Damage also extended ventrally into the cingulum and corpus callosum bilaterally. In one case, there was unilateral involvement of lateral CA1, and in another similar damage was seen bilaterally. $\Lambda t$ the level of the hippocampal flexure, the only hippocampal damage in evidence involved unilateral damage to the CA1 field in two animals. All control lesions, however, caused bilateral elevation of the hippocampal tissue subjacent to the lesions, where this tissue filled the cavity created by aspiration of overlying cortex.

\section{RESULTS}

During training, the mean number of days needed to reach criterion on Problems 1-3 (the first through third discrimination problems encountered by animals) was $17.00 \pm 1.70,11.20 \pm 1.93$, 


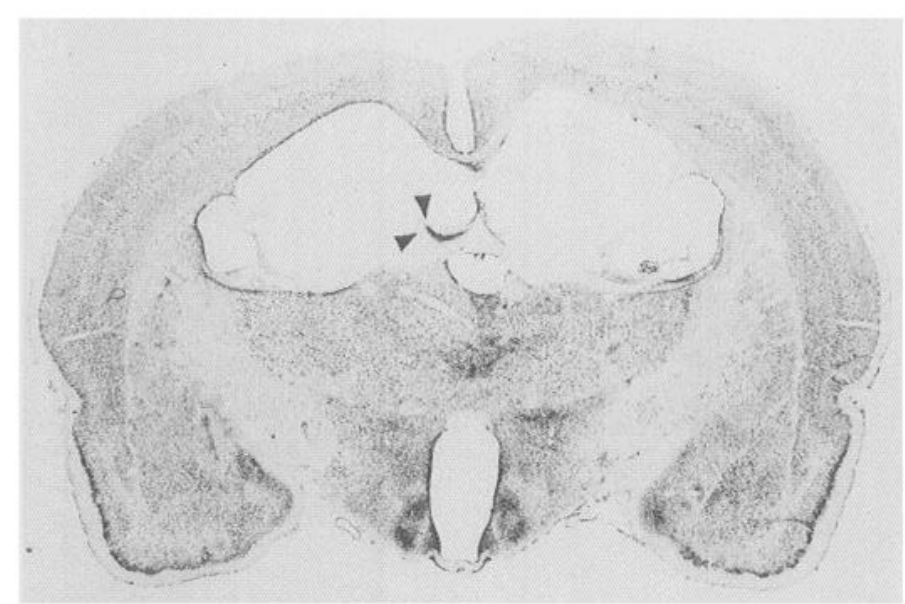

Figure 2. Photomicrograph of a coronal section from a rat with bilateral dorsal hippocampal lesions. In this cresyl violet-stained section, rostral to the aspiration-cannula insertion site, the entire hippocampus has been removed except for a small segment of dentate gyrus on the left side (indicated by arrowheads). Histological material from most animals demonstrated complete or near complete elimination of dorsal hippocampus at rostral levels, with more variable sparing caudally.

and $12.60 \pm 2.54$, respectively, for the NC group, and $15.00 \pm$ $2.83,13.25 \pm 4.03$, and $7.25 \pm 1.60$ for the DHC group. The two groups also showed similar levels of performance during the retraining phase of testing, as $\mathrm{NC}$ animals achieved means of 3.20 $\pm 0.37,4.00 \pm 1.00$, and $4.60 \pm 1.60$ days-to-criterion on Problems $1-3$, and DHC animals $2.50 \pm 0.96,4.50 \pm 1.26$, and $5.75 \pm 0.85$ on these problems (in the same order). All of the statistical analyses to follow were first performed on the behavioral data of animals in the NC and DHC groups, which confirmed that these two control groups performed comparably on the object discrimination problems. Because none of the analyses indicated a significant difference between the groups, or interactions with a group factor, the data from NC and DHC animals were pooled and treated as a single data set. This data set will hereafter be referred to as the CON group.

There was a $15 \mathrm{~d}$ limit on testing for each problem during retraining. This was done to control the interval between training and retraining on problems that followed one with which an animal struggled. Three DH animals could not reach criterion on one or more problems by this limit, and a score of 15 was given for each.

A $p$ level of 0.05 was set for all statistical analyses.

As illustrated in Figure 3, on the training phase of object discrimination testing DH animals had means of $14.10 \pm 1.97$, $12.78 \pm 2.43$, and $7.67 \pm 1.37$ on Problems 1,2 , and 3 respectively, which was similar to the performance of control rats; $\mathrm{CON}$ animals had means of $16.11 \pm 1.50,12.11 \pm 1.97$, and $10.22 \pm 1.76$. The performance of lesioned and control rats, however, diverged during retraining, as the $\mathrm{DH}$ group required more days to achieve criterion performance on the discrimination problems than the CON group. During retraining, the mean number of days-tocriterion for the DH group was, in order, $7.89 \pm 1.30,8.56 \pm 1.65$, and $7.89 \pm 1.95$ on Problems $1-3$, whereas for the CON group means of $2.89 \pm 0.45,4.22 \pm 0.74$, and $5.11 \pm 0.93$ were achieved (Fig. 4).

A 2 (group; $\mathrm{DH}$ vs $\mathrm{CON}) \times 3$ (problem order; Order 1 vs Order 2 vs Order 3) $\times 3$ (problem; Problem 1 vs Problem 2 vs Problem 3) $\times 2$ (testing phase; training vs retraining) repeated-measures $\exists$ Dorsal Hippocampus $\square$ Control

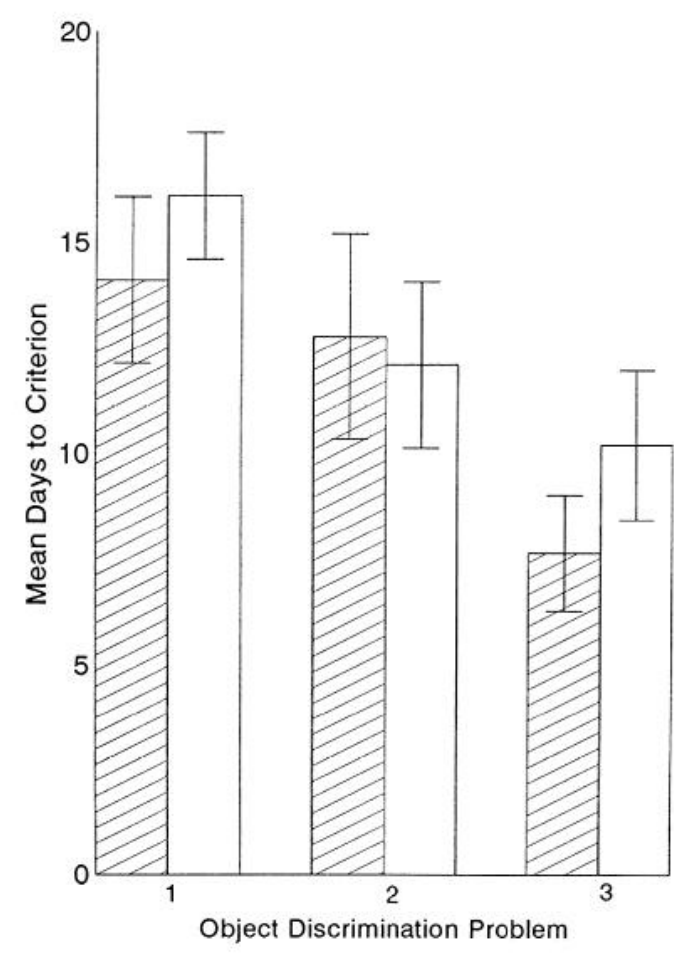

Figure 3. Performance on the training (acquisition) phase of object discrimination testing. Dorsal hippocampal (hatched bars) and control animals (open bars) did not differ on the number of days of testing needed to reach criterion on the three discrimination problems. Error bars depict SEM.

ANOVA confirmed that a difference between $\mathrm{DH}$ and $\mathrm{CON}$ animals was exclusive to performance during retraining. Although the main effect of group was not significant $\left(F_{(1,12)}=0.69\right)$, a significant interaction between group and testing phase was evident $\left(F_{(1,12)}=9.32, p<0.05\right)$. Thus, whereas rats with dorsal hippocampal lesions and controls did not differ significantly overall on this task, a difference between groups at one of the testing phases was in evidence. Analysis of the simple effects of this interaction revealed that whereas lesioned and control animals performed similarly during the training (acquisition) phase of testing, controls reached criterion on the problems more quickly than dorsal hippocampal rats during retraining (retention). During training, $\mathrm{DH}$ animals achieved an overall (across problems) mean of $11.52 \pm 1.53 \mathrm{~d}$ to reach criterion on each problem, which did not differ from the mean of $12.81 \pm 1.05 \mathrm{~d}$ found for the CON group $\left(F_{(1,12)}=0.52\right)$. In contrast, during retraining the overall mean of $8.11 \pm 1.33 \mathrm{~d}$ for DH rats was found to be significantly greater than the CON mean of $4.07 \pm 0.35\left(F_{(1,12)}=9.67, p<\right.$ $0.01)$. For the other between-subjects factor, problem order, neither a main effect $\left(F_{(2,12)}=0.57\right)$ nor a group $\times$ order interaction $\left(F_{(2,12)}=0.97\right)$ was found.

The ANOVA also revealed a main effect of testing phase, which indicated that, across groups and problems, fewer days of testing were required to reach criterion during retraining $(6.09 \pm 0.58)$ than during training $(12.17 \pm 0.81)\left(F_{(1.12)}=46.83, p<0.01\right)$. This reduction in days-to-criterion suggests that performance during retraining benefitted from the acquisition of information during training, which can be ascribed to memory of the discrimina- 


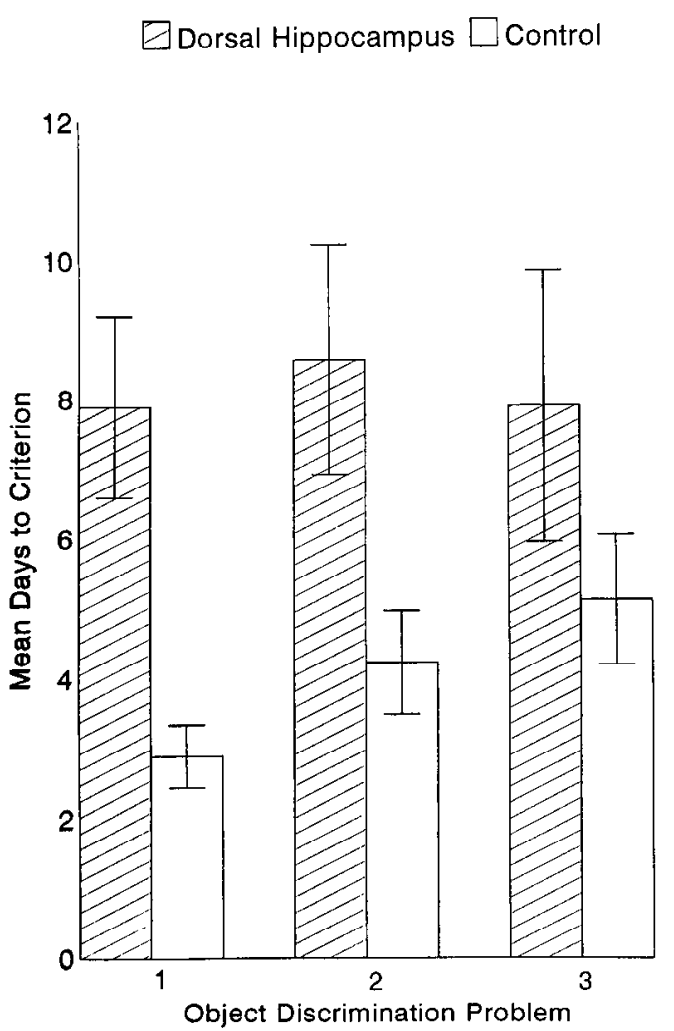

Figure 4. Performance on the retraining (retention) phase of object discrimination testing. Dorsal hippocampal (hatched bars) animals reached criterion in significantly more days than controls (open bars) on the three discrimination problems during retraining. Error bars depict SEM.

tion problems. Consistent with this main effect, further simple effects analyses on the group $\times$ testing phase interaction described above revealed significant improvement-and therefore retention-in performance by both the lesioned and control groups during retraining. The mean days-to-criterion of the CON group during retraining $(4.07 \pm 0.35)$ was significantly lower than the mean of this group at training $(12.81 \pm 1.05)\left(F_{(1,12)}=47.09\right.$, $p<0.01)$, and the DH mean at retraining $(8.11 \pm 1.33)$ was also significantly lower than that of training $(11.52 \pm 1.53)\left(F_{(1,12)}=\right.$ $7.48, p<0.05)$.

In addition, a main effect of problem was found $\left(F_{(2,24)}=4.30\right.$, $p<0.05$ ), which a Sheffe's test revealed was attributable to a significant difference between performance on Problem 1 (10.25 $\pm 1.11)$ and Problem $3(7.72 \pm 0.80)$, although neither of these problems differed from Problem $2(9.42 \pm 1.04)$. This trend implies that there was overall improvement on the object discrimination problems as testing progressed, probably because procedural aspects of testing were mastered with experience. This expertise, however, appears to have been acquired gradually, such that incremental gains in performance across all problems was not evident and significant improvement was obtained only when the first and last problems were compared.

To determine whether performance was affected by the correction procedure used in the present behavioral paradigm, analyses were conducted on the number of responses needed to reach criterion on the discrimination problems. Animals were given 20 trials of training in each testing session, but each trial was repeated until a correct response was given (see Procedure). An

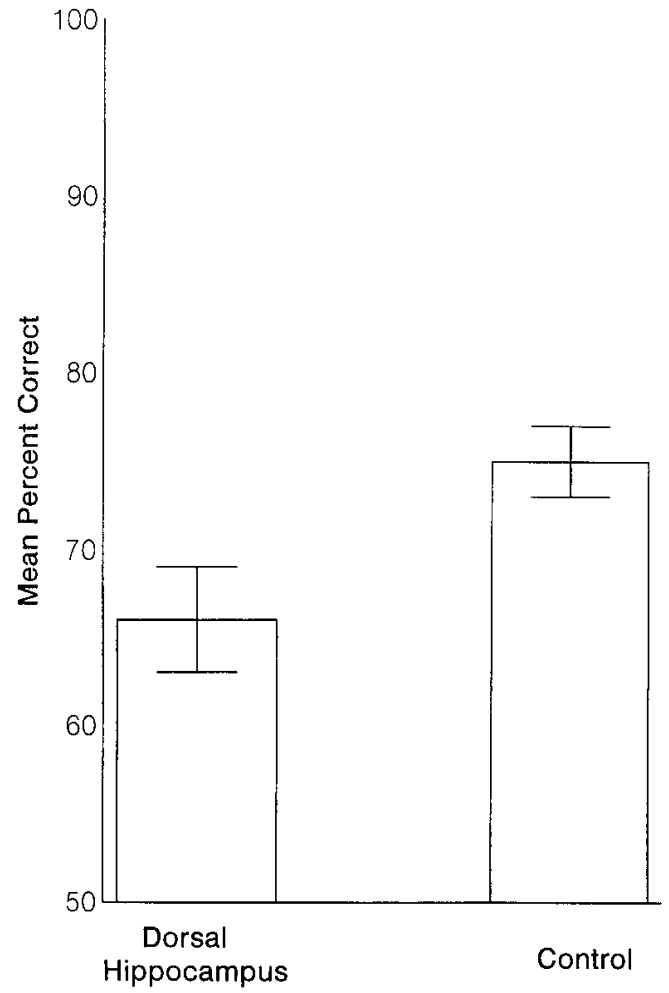

Figure 5. Average percent of correct responses on the first day of retraining for all discrimination problems. Overall, control animals (right) made significantly more correct responses on the first day of testing during retraining than dorsal hippocampal animals (left). Error bars depict SEM.

analysis of the total number of responses-to-criterion, therefore, accounts for the correction procedure in the evaluation of group performance on the discrimination problems. A 2 (group) $\times 3$ (problem order) $\times 3$ (problem) $\times 2$ (testing phase) repeatedmeasures ANOVA revealed the same pattern of results as that found for the days-to-criterion dependent variable, thereby confirming the acquisition/retention dissociation seen in the $\mathrm{DH}$ group.

Also of interest is the degree to which the representations of the discrimination problems were established at the onset of the retention phase of testing, rather than learned again during retraining. An index of this construct can be obtained by assessing performance on the first day of testing for each problem during retraining. The CON group achieved means of $15.89 \pm 0.86,14.33$ \pm 1.05 , and $14.67 \pm 0.94$ correct responses on Problems $1-3$, for an overall mean of $14.96 \pm 0.49$ and an average accuracy of $74.80 \%$. The DH group, in contrast, was found to have means of $12.89 \pm 0.79,13.00 \pm 0.97$, and $13.56 \pm 0.78$ correct responses on the discrimination problems, in order, for an overall mean of $13.15 \pm 0.53$ correct responses on day 1 and an average accuracy of $65.75 \%$ (Fig. 5). A 2 (group) $\times 3$ (discrimination problem) repeated-measures ANOVA was performed on these data and found an effect of group $\left(F_{(1,16)}=6.37, p<0.05\right)$. This analysis reveals that control animals made significantly more correct responses than hippocampal animals even on the first day of retraining on the discrimination problems. Thus, the retention deficit that hippocampal rats suffered is evident even at the outset of retraining on each problem. Although $\mathrm{DH}$ animals were found to be impaired on day 1 performance, the average accuracy of the 
group $(65.75 \%)$ is suggestive of above-chance memory and, hence, of significant retention. To determine whether this suggestion is accurate, a $t$ test was performed on day 1 retraining performance for all problems, comparing the mean number of correct responses given by $\mathrm{DH}$ rats to chance performance (i.e., 10 correct responses). These analyses confirmed that the $\mathrm{DH}$ group performed above-chance on the first day of retraining on all three problems and, hence, showed significant retention of the problems (Problem 1: $t_{(8)}=3.66, p<0.01$; Problem 2: $t_{(8)}=3.09$, $p<0.05$; Problem 3: $t_{(8)}=4.54, p<0.01$ ).

To determine whether the impaired retention seen in hippocampal animals can be attributed to the individual pairs of objects used as discrimination problems in the present behavioral paradigm, performance on the actual object pairs, independent of position in testing order, was subjected to a 2 (group) $\times 3$ (problem order) $\times 3$ (object pair) $\times 2$ (testing phase) repeatedmeasures ANOVA. This analysis failed to find an interaction between group and object pair $\left(F_{(2,24)}=1.02\right)$ or any three-way or four-way interactions involving these factors (all $F<2.00$ ). Thus, neither the DH nor the CON group was differentially affected by the individual object pairs used to test acquisition and retention in the present behavioral task.

\section{DISCUSSION}

The central finding of the present study is that lesions of the dorsal hippocampus selectively impaired the retention of object discrimination problems over a 3 week delay interval. Rats with dorsal hippocampal injury were normal at acquiring three discrimination problems but showed compromised memory of this information during the retention phase of testing, consistent with a consolidation view of hippocampal function (Squire, 1987, 1992). This acquisition/retention dissociation is similar to that seen in rats with entorhinal-hippocampal disconnection from angular bundle transections (Vnek et al., 1995). These consonant results argue for a role in long-term object memory for the hippocampal formation, but one that is limited to the retention of information.

There is precedence for the selective involvement of entorhinal cortex in long-term retention on other discrimination tasks. For example, rats with lesions of the entorhinal cortex have shown normal acquisition but impaired retention, after a $10 \mathrm{~d}$ delay, of a brightness discrimination (Levisohn and Isacson, 1991). Additionally, entorhinal cortex-lesioned rats have demonstrated normal acquisition of an olfactory discrimination, but impaired performance when called upon to retain this information over delays ranging from 1 to $3 \mathrm{hr}$ (Staubli et al., 1984, 1986). Together with the present findings and those from rats with angular bundle lesions (Vnek et al., 1995), these results indicate that the hippocampus and entorhinal cortex are particularly important for the long-term retention of a variety of different types of information.

The performance of rats with dorsal hippocampal lesions during the training phase of testing fits into a long history of studies showing that animals with limbic system injury can normally acquire visual discrimination problems. Despite hippocampal and/or parahippocampal damage, both monkeys and rats have consistently demonstrated a preserved capacity to learn single object and pattern discrimination problems (Mishkin, 1954; Orbach et al., 1960; Correll and Scoville, 1965; Moss et al., 1981; Zola-Morgan and Squire, 1986; Zola-Morgan et al., 1989; Wible et al., 1992; Wood et al., 1993; Alvarez et al., 1995). The present findings add to this body of work by showing that large hippocampal lesions are not deleterious to the acquisition of a list of object discrimination problems.
Our findings also accord with the performance of patient $\mathrm{H}$. M. on measures of long-term picture recognition. As noted earlier, when given extra time to study stimuli $(15-20 \mathrm{sec}) \mathrm{H}$. M. can demonstrate normal memory on a yes/no test of picture recognition even after a delay interval as long as 1 day. However, when delays are extended to one week or more, H. M.'s accuracy falls well below that of controls (Huppert and Piercy, 1979; Freed and Corkin, 1988). Although a test of picture memory differs markedly from the behavioral paradigm used in the present study, these tasks do bear some resemblance to each other in that they are both indices of visual recognition memory. It is rather compelling, therefore, that a visual recognition deficit is seen in $\mathrm{H}$. M. and in rats with hippocampal damage only after relatively long (i.e., 1 week or more) delays are imposed between acquisition and a test of retention.

The present results have important implications for the debate over hippocampal involvement in nonspatial memory. This debate stems from studies of animals with restricted hippocampal lesions that have been found to perform normally on measures of nonspatial memory. For example, Jarrard (1989) has developed a protocol using ibotenic acid that causes highly selective, axonsparing lesions of the hippocampus in rats. Preliminary studies have found that rats with this lesion are not impaired on concurrent object discrimination (COD) (Jarrard and Hyko, 1994) and single-object discrimination learning (Astur et al., 1994). In the monkey, Alvarez et al. (1995) used magnetic resonance imaging scans to determine the sites of electrode placements in the production of radio-frequency lesions. This strategy also produces discrete hippocampal lesions, and lesioned monkeys perform normally on eight-pair COD, pattern discrimination, and singleobject discrimination learning (Alvarez et al., 1995).

The normal memory demonstrated by both monkeys and rats with selective lesions of the hippocampus on various nonspatial behavioral tasks has called into question the role of the hippocampus in memory. Some have argued that rhinal cortex is the medial temporal area most critical for memory (Meunier et al., 1993; Mumby and Pinel, 1994), and others have maintained that the hippocampus is selectively involved in spatial memory functions [see Amaral and Witter (1991) for a discussion of this issue]. Findings from the studies of nonspatial memory described above, however, cannot be considered conclusive because they only measured one aspect of memory, information acquisition. Thus, if the hippocampus is in fact selectively involved in the retention of nonspatial information, as the present and our earlier results (Vnek et al., 1995) suggest, then behavioral tasks that do not include a measure of retention may be insensitive assays of hippocampal function. Failure to find deficits on measures of nonspatial memory in animals with selective hippocampal lesions may well be attributable to the limited scope of the behavioral tasks used to assess memory in these studies.

Behavioral paradigms that assess both acquisition and retention allow for analyzing delay effects with experimental animals, an important parameter in human memory research. Studies of human clinical populations, as well as studies of normal human memory, often examine the integrity of information (i.e., forgetting) after delays ranging from minutes to weeks (see Haist et al., 1992). In contrast, tasks used to assess forgetting in lesioned animals usually include a far more limited range of delays. For example, in work with animals the paradigm most commonly used to examine nonspatial working memory is delayed nonmatchingto-sample, and although varying delay intervals is an integral part of this task, delays in excess of $40 \mathrm{~min}$ are not used (c.g., Alvarez 
et al., 1995). Thus, measuring delay intervals that vary on the order of days rather than minutes in work with animals offers the prospect of coalescing research of human amnesic syndromes and paradigms that model them in experimental animals.

There is now considerable evidence that the cortices of the parahippocampal region (i.e., entorhinal, perirhinal, and parahippocampal) play an important role in memory (for review, see Murray, 1992; Squire, 1992; Zola-Morgan and Squire, 1993; Mishkin and Murray, 1994). This position derives from studies using selective lesions of the parahippocampal region in experimental animals. Both monkeys (Zola-Morgan et al., 1989; Gaffan and Murray, 1992; Meunier et al., 1993; Suzuki et al., 1993) and rats (Otto and Eichenbaum, 1992; Bunsey and Eichenbaum, 1993; Mumby and Pinel, 1994; Nagahara et al., 1995) with lesions restricted to one or more of the cortices of the parahippocampal region demonstrate severe anterograde amnesia. Thus, the contribution of cortices adjacent to the hippocampus must be considered when behavioral deficits follow hippocampal damage. Damage to these cortices can be incurred either directly while lesioning the adjacent hippocampus or indirectly from degeneration of afferents arising from rhinal cortex after removal of their hippocampal targets (Peterson et al., 1994).

In the present study, dorsal hippocampal lesions were used rather than complete removal of the hippocampus to ensure that rhinal cortex, which is principally adjacent to ventral hippocampus in the rat, would be spared in all animals. However, dorsal hippocampal lesions were large and likely led to degeneration of cells in layer II of entorhinal cortex, the source of perforant path projections to the hippocampus. Thus, the contribution of entorhinal cortex to the retention deficit found in dorsal hippocampal rats cannot be ruled out.

Although it is not clear whether the retention deficit found in dorsal hippocampal rats can be ascribed to the hippocampus, entorhinal cortex, or a combination of the two, it appears that these structures were not critical for the acquisition of this information. As for what system supported acquisition in lesioned animals, a corticostriatal circuit is a plausible candidate. This system is thought to govern the acquisition of habits which, in contrast to declarative memories, are "noncognitive stimulusresponse bonds" (Mishkin and Petri, 1984). Acquisition of the three discrimination problems may have amounted to establishing a stimulus-response association between each correct object-or each pair of objects-and the (object) displacement response, which was ostensibly bound by the food reinforcement. Alternatively, the normal acquisition demonstrated by rats with dorsal hippocampal lesions may have been mediated by rhinal cortex, which was not directly affected by hippocampal lesions. As noted above, there is a wealth of empirical data implicating the rhinal cortex in nonspatial memory; thus, this area may have been the neuroanatomical site that subserved the capacity for acquisition in both normal and lesioned rats.

To conclude, dorsal hippocampal lesions-or secondary damage to hippocampal afferents in entorhinal cortex-do not affect the acquisition of multiple, successively learned discrimination problems but can impair the retention of this information over a long delay interval. This corroborates our earlier finding that entorhinal-hippocampal disconnection also selectively impairs retention on the behavioral paradigm used in the present study (Vnek et al., 1995). An intact hippocampus and/or its afferents in entorhinal cortex, therefore, appear to be necessary for long-term retention, even if these structures were not involved in acquiring the information.

\section{REFERENCES}

Alvarez P, Zola-Morgan S, Squire LR (1995) Damage limited to the hippocampal region produces long-lasting memory impairment in monkeys. J Neurosci 15:3796-3807.

Amaral DG, Witter MP, editors (1991) Is the hippocampal formation preferentially involved in spatial behavior? Hippocampus 1:221-340.

Astur RS, Mumby DG, Weisend MP, Sutherland RJ (1994) Hippocampal damage in rats causes retrograde amnesia for place navigation but not for object discriminations. Soc Neurosci Abstr 20:1015.

Braak H, Braak E (1993) Entorhinal-hippocampal interaction in mnestic disorders. Hippocampus 3:239-246.

Bunsey M, Eichenbaum H (1993) Critical role of the parahippocampal region for paired-associate learning in rats. Behav Neurosci 107:740-747.

Correll RE, Scoville WB (1965) Effects of medial temporal lesions on visual discrimination performance. J Comp Physiol Psychol 60:175-181.

Freed DM, Corkin S (1988) Rate of forgetting in H. M.: 6-month recognition. Behav Neurosci 102:823-827.

Gaffan D, Murray EA (1992) Monkeys (macaca fascicularis) with rhinal cortex ablations succeed in object discrimination learning despite $24 \mathrm{hr}$ intertrial intervals and fail at matching to sample despite double sample presentations. Behav Neurosci 106:30-38.

Haist F, Shimamura AP, Squire LR (1992) On the relationship between recall and recognition memory. J Exp Psychol [Hum Learn] 18:691-702.

Huppert FA, Piercy M (1979) Normal and abnormal forgetting in organic amnesia: effect of locus of lesion. Cortex 15:385-390.

Jarrard LE (1989) On the use of ibotenic acid to lesion selectively different components of the hippocampal formation. J Neurosci Methods 29:251-259.

Jarrard LE (1993) On the role of the hippocampus in learning and memory in the rat. Behav Neural Biol 60:9-26.

Jarrard L, Hyko A (1994) Complementary roles for the hippocampus and entorhinal/perirhinal cortex in the acquisition of spatial and nonspatial tasks in the rat. Soc Neurosci Abstr 20:1015.

Levisohn LF, Isacson O (1991) Excitotoxic lesions of the rat entorhinal cortex. Effects of selective neuronal damage on acquisition and retention of a non-spatial reference memory task. Brain Res 564:230-244.

Meunier M, Bachevalier J, Mishkin M, Murray EA (1993) Effects on visual recognition of combined and separate ablations of the entorhinal and pcrirhinal cortex in rhesus monkeys. J Neurosci 13:5418-5432.

Mishkin M (1954) Visual discrimination performance following partial ablations of the temporal lobe. II. Ventral surface vs. hippocampus. J Comp Physiol Psychol 47:187-193.

Mishkin M, Murray EA (1994) Stimulus recognition. Curr Opin Neurobiol 4:200-206.

Mishkin M, Petri HL (1984) Memories and habits: some implications for the analysis of learning and retention. In: Neuropsychology of memory (Squire LR, Butters N, eds), pp 287-296. New York: Guilford.

Moser E, Moser M-B, Andersen P (1993) Spatial learning impairment parallels the magnitude of dorsal hippocampal lesions, but is hardly present following ventral lesions. J Neurosci 13:3916-3925.

Moser M-B, Moser EI, Forrest E, Andersen P, Morris RGM (1995) Spatial learning with a minislab in the dorsal hippocampus. Soc Neurosci Abstr 21:1942.

Moss M, Mahut H, Zola-Morgan S (1981) Concurrent discrimination learning of monkeys after hippocampal, entorhinal, or fornix lesions. J Neurosci 1:227-240.

Mumby DG, Pinel JPJ (1994) Rhinal cortex lesions and object recognition in rats. Behav Neurosci 108:11-18.

Murray EA (1992) Medial temporal lobe structures contributing to recognition memory: the amygdaloid complex versus the rhinal cortex. In: The amygdala: neurobiological aspects of emotion, memory, and mental dysfunction (Aggleton JP, ed), pp 453-470. New York: Wiley.

Nagahara AH, Otto T, Gallagher M (1995) Entorhinal-perirhinal lesions impair performance of rats on two versions of place learning in the Morris water maze. Behav Neurosci 109:3-9.

Orbach J, Milner B, Rasmussen T (1960) Learning and retention in monkeys after amygdala-hippocampus resection. Arch Neurol 3:230-251.

Otto T, Eichenbaum H (1992) Complementary roles of the orbital prefrontal cortex and the perirhinal-entorhinal cortices in an odor-guided delayed-nonmatching-to-sample task. Behav Neurosci 106:762-775.

Paxinos G, Watson C (1986) The rat brain in stereotaxic coordinates. 2nd Ed. San Diego: Academic.

Peterson DA, Lucidi-Phillipi CA, Eagle KL, Gage FH (1994) Perforant path damage results in progressive neuronal death and somal atrophy in 
layer II of entorhinal cortex and functional impairment with increasing postdamage age. J Neurosci 14:6872-6885.

Scoville WB, Milner B (1957) Loss of recent memory after bilateral hippocampal lesions. J Neurol Neurosurg Psychiatry 20:11-21.

Squire LR (1987) Memory and brain. New York: Oxford UP.

Squire LR (1992) Memory and the hippocampus: a synthesis from findings with rats, monkeys, and humans. Psychol Rev 99:195-231.

Staubli U, Ivy G, Lynch G (1984) Hippocampal denervation causes rapid forgetting of olfactory information in rats. Proc Natl Acad Sci USA $81: 5885-5887$.

Staubli U, Fraser D, Kessler M, Lynch G (1986) Studies on retrograde and anterograde amnesia of olfactory memory after denervation of the hippocampus by entorhinal cortcx lesions. Behav Neural Biol 46:432-444.

Suzuki WA, Zola-Morgan S, Squire LR, Amaral DG (1993) Lesions of the perirhinal and parahippocampal cortices in the monkey produce long-lasting memory impairment in the visual and tactual modalities. J Neurosci 13:2430-2451.

Vnek N, Gleason TC, Kromer LF, Rothblat LA (1995) Entorhinalhippocampal connections and object memory in the rat: acquisition versus retention. J Neurosci 15:3193-3199.

Wible CG, Shiber JR, Olton DS (1992) Hippocampus, fimbria-fornix, amygdala, and memory: object discriminations in rats. Behav Neurosci 106:751-761.
Wickelgren WA (1968) Sparing of short-term memory in an amnesic patient: implications for strength theory of memory. Neuropsychologia 6:235-244.

Wood ER, Mumby DG, Pinel JPJ, Phillips AG (1993) Impaired object recognition memory in rats following ischemia-induced damage to the hippocampus. Behav Neurosci 107:51-62.

Zola-Morgan S, Squire LR (1985) Medial temporal lesions in monkeys impair memory on a variety of tasks sensitive to human amnesia. Behav Neurosci 99:22-34.

Zola-Morgan S, Squire LR (1986) Memory impairment in monkeys following lesions limited to the hippocampus. Behav Neurosci 100:155-160.

Zola-Morgan S, Squire LR (1993) Neuroanatomy of memory. Annu Rev Neurosci 16:547-563.

Zola-Morgan S, Squire LR, Amaral DG (1986) Human amnesia and the medial temporal region: enduring memory impairment following a bilateral lesion limited to field CAl of the hippocampus. J Neurosci 6:2950-2967.

Zola-Morgan S, Squire LR, Amaral DG, Suzuki WA (1989) Lesions of perirhinal and parahippocampal cortex that spare the amygdala and hippocampal formation produce severe memory impairment. J Neurosci 9:4355-4370. 